\title{
Genetic heterogeneity in autosomal dominant essential tremor
}

\author{
Margaret J. Kovach, $P h D^{* 1}$, Jimmy Ruiz, $B S^{* 1}$, Katerina Kimonis ${ }^{1}$, Sajjad Mueed, $M D^{2}$, Shobhit Sinha, $M D^{2}$, \\ Connie Higgins, $M A^{2}$, Suzanne Elble, $M A^{2}$, Rodger Elble, $M D, P h D^{2}$, and Virginia E. Kimonis, $M D, M R C P^{1}$
}

\begin{abstract}
Purpose: To perform linkage analysis of candidate loci in a large Midwestern family with autosomal dominant essential tremor. Methods: Thirty-eight members of a six-generation family were evaluated for essential tremor using consensus criteria. Linkage analysis was performed with microsatellite markers reported for three genetic loci associated with familial essential tremor. Results: Patients exhibited a combination of postural and kinetic tremor involving primarily the arms and hands, with a mean age of onset of 31 years. Genetic studies excluded linkage to ETM1 and ETM2 loci, as well as a candidate locus for parkinsonism and postural tremor on chromosome $4 p$. Conclusion: Familial essential tremor is a common hereditary movement disorder demonstrating phenotypic variability and genetic heterogeneity. Genetics in Medicine, 2001:3(3):197-199.
\end{abstract}

Key Words: familial essential tremor, autosomal dominant, linkage analysis

Essential tremor (ET) is the most common movement disorder in humans, affecting $0.3-1.7 \%$ of the population. ${ }^{1}$ It affects people of all ages and ethnic groups, but its prevalence increases with age, affecting at least $5 \%$ of people age 65 or older. ${ }^{2,3}$ It is a progressive disorder exhibiting variable expression with symptoms ranging from a mild to coarse tremor characteristically affecting the upper limbs with involvement of the head, facial muscles, voice, and lower extremities in some individuals. ${ }^{4}$ ET can become quite debilitating due to severe disfiguring head and postural tremor as well as a disabling loss of speech and fine motor hand control and accompanying psychosocial problems. Beta-blockers such as propranolol and primidone have limited effectiveness, and thalamotomy and thalamic deep-brain stimulation are expensive surgical procedures with a $9-23 \%$ risk of permanent complications, including a $1-4 \%$ risk of intracerebral hemorrhage. ${ }^{5}$

Variability in diagnostic criteria, incomplete penetrance, and high phenocopy rate have complicated genetic studies of ET. However, it is generally considered that approximately $50 \%$ of ET cases are familial and are inherited in an autosomal dominant fashion. Previous studies of autosomal dominant pedigrees of ET have identified two disease loci, ETM1 (MIM 190300) ${ }^{6}$ and ETM2 (MIM 602134). ${ }^{6}$ In a study of 75 affected

\footnotetext{
From the ${ }^{1}$ Division of Genetics and Metabolism, Department of Pediatrics, Southern Illinois University School of Medicine, Springfield, Illinois; and ${ }^{2}$ Department of Neurology, Southern Illinois University School of Medicine, Springfield, Illinois.

${ }^{*}$ These individuals contributed equally to this work.

Virginia E. Kimonis, MD, MRCP, Division of Genetics and Metabolism, Department of Pediatrics, Southern Illinois University School of Medicine, PO Box 19658, Springfield, IL 62794-9658; E-mail: vkimonis@siumed.edu.

Received: January 4, 2001

Accepted: February 19, 2001
}

individuals from 16 Icelandic families, the gene for familial ET (FET1 or ETM1) was mapped to chromosome $3 \mathrm{q} 13$ with a maximized overall LOD score $>3.7 .7$ The LOD scores, obtained from independent analysis of the families, ranged from -0.720 to 1.290 (penetrance of 0.90 ; phenocopy rate of 0.10 ), with 10 of 16 families contributing positive LOD scores. A second locus, ETM2, on chromosome 2p22-p25, was subsequently identified by linkage analysis of a large family of Czech descent. ${ }^{8}$ Later, Higgins et al. ${ }^{9}$ presented evidence for linkage of three unrelated American families with ET to the ETM2 locus. Recently, a chromosome $4 \mathrm{p}$ haplotype was reported for Lewy body parkinsonism (LBP) that also segregated among individuals in the pedigree who exhibited only postural tremor consistent with $\mathrm{ET}^{10}$ (MIM 168601). ${ }^{6}$

Additional-as yet unidentified-loci of this trait are indicated in families excluding linkage to these reported loci. ${ }^{11}$ This current report describing a large Midwestern American family presenting with familial ET supports further genetic heterogeneity of familial ET. Linkage analysis of this family determined that the disease is genetically distinct from familial ET on chromosomes 2 and 3, as well as LBP on chromosome 4.

Members of the six-generation Midwestern family (Fig. 1) presenting with familial ET participated after giving their informed consent, approved by the Springfield Committee for Research Involving Human Subjects. Members of this large family were examined by a neurologist and diagnosed with consensus criteria. ${ }^{12}$

The amplitude of tremor was quantified with the following ordinal rating scale: $0=$ no tremor; $1+=$ slight (barely noticeable) tremor; $2+=$ moderate, readily noticeable tremor $(<1 \mathrm{~cm}$ excursions); $3+=$ marked, partially disabling tremor (1-2 cm excursions); $4+=$ severe, coarse, disabling tremor ( $>2 \mathrm{~cm}$ excursions). Tremor in each patient was diagnosed as 


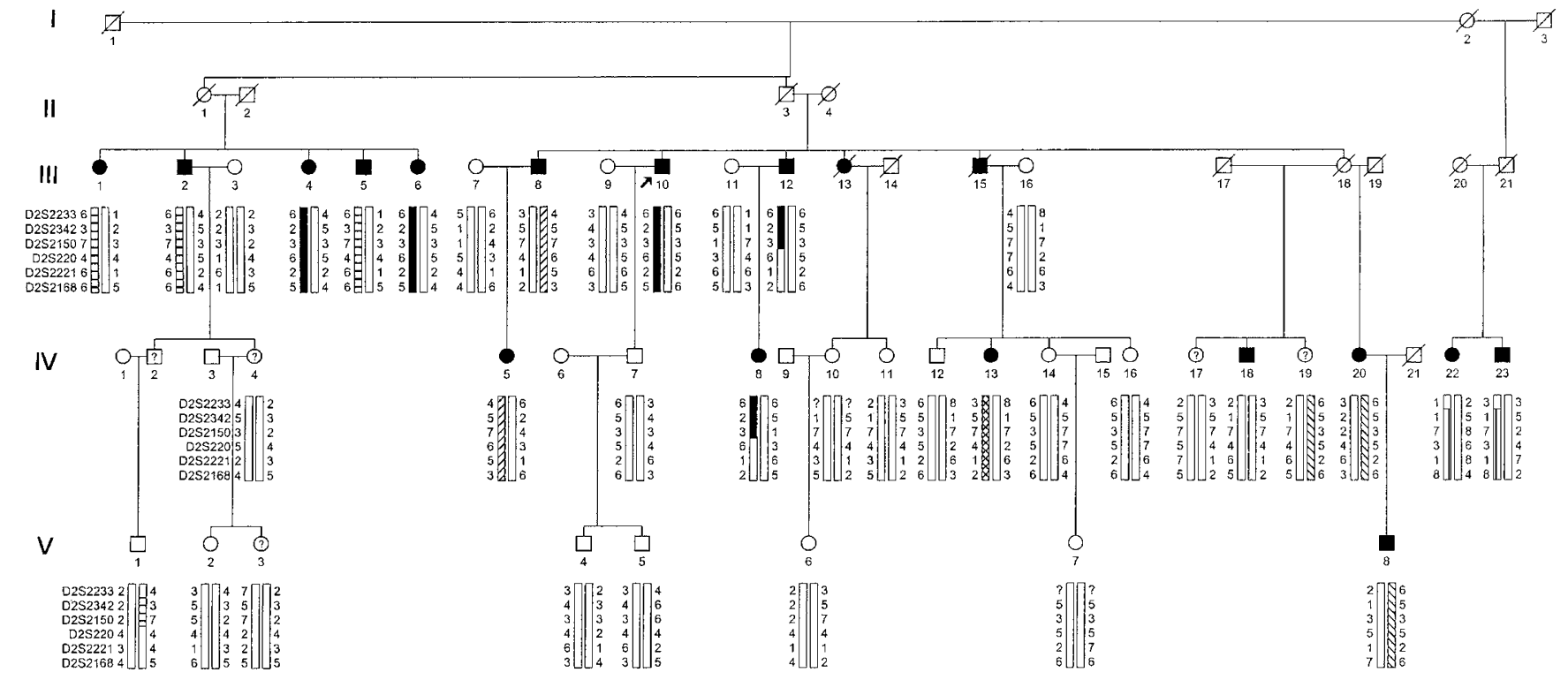

Fig. 1 Partial pedigree of family affected with autosomal dominant essential tremor showing haplotypes of chromosome 2p25-p22. The markers are listed in order from centromere to telomere. Disease haplotypes were inferred among nuclear families within the whole pedigree and are indicated by patterned bars. Five different haplotypes were determined among affected individuals, indicating that the disease is not linked to chromosome $2 \mathrm{p}$. Squares represent males and circles represent females. Shaded symbols denote individuals with essential tremor and a "?" denotes individuals with unknown affection status. The proband is indicated by an arrow.

definite, probable, or possible, according to the following criteria:

- Definite ET: bilateral arm tremor with 2+ amplitude in at least one arm or predominant cranial-cervical tremor with $2+$ amplitude and at least $1+$ tremor in at least one arm.

- Probable ET: $1+$ arm tremor bilaterally or isolated $2+$ cranial-cervical tremor or convincing history of ET.

- Possible ET: isolated 1+ cranial-cervical tremor or taskor position-specific upper-limb tremor or unilateral arm tremor.

Genomic DNA was extracted using the PUREGENE DNA Isolation Kit (Gentra Systems, Minneapolis, MN). The concentration was estimated by standard techniques. Linkage analysis was performed with microsatellite markers that resulted in significant LOD scores for the previously reported loci for familial ET on chromosomes 2 and 3, as well as LBP: D3S1278, D3S1558, D3S3515, and D3S1267 for ETM1; D2S2233, D2S2150, D2S2342, D2S2221, and D2S2168 for ETM2; D4S230 and D4S1609 for LBP.

Amplification was conducted with forward primers tagged with fluorescent dyes (Research Genetics, Inc., Huntsville, AL). Unlabeled primers were tagged with fluorescein using a two-step method ${ }^{13}$ in which a phosphorothioate group is transferred to the 5'-hydroxyl group of the oligonucleotide in a reaction catalyzed by $\mathrm{T}_{4}$ polynucleotide kinase using adenosine 5'-O-3-thiotriphosphate (ATP $\gamma S$; Sigma, St. Louis, MO) as substrate. In the second step the phosphorothioate oligonucleotide is reacted with 5-iodoacetamidofluorescein (5-IAF; Molecular Probes, Eugene, OR). Amplification products were analyzed with an FMBIO-100 fluorescent image scanning unit
(Hitachi, South San Francisco, CA). Alleles were scored, and genotype data were entered into the pedigree file of the LINKAGE computer package.

A standard LOD score approach using the MLINK option of the software LINKAGE package was for 2-point linkage analysis. ${ }^{14}$ Familial ET was modeled as an autosomal dominant trait allowing for age-specific reduced penetrance and phenocopies. A disease allele frequency of 0.01 was assumed. The following penetrance model was used for the disease genotype: $\mathrm{dd}, \mathrm{Dd}$, and DD, where $\mathrm{D}$ is the disease allele. Four age-dependent risk classes were used in the analysis: Class I ( $0-20$ years), 0.00001, 0.001, 0.001; Class II (21-30 years), 0.00005, 0.15, 0.15 ; Class III (31-40 years), 0.002, 0.30, 0.30; and Class IV (>40 years), $0.01,0.90,0.90$.

DNA was obtained from 38 members of the family, in which 16 were definitely affected by ET. The age in which tremor was first identified appeared to be earlier in each generation. The mean ages of onset were 43 years in generation III $(N=8$; range 15-69 years), 32 years in generation IV $(N=7$; range $12-51$ years), and 6 years in generation $\mathrm{V}(N=1)$.

Linkage analysis was carried out with molecular markers for established loci of autosomal dominant ET (Table 1). The ETM1 locus has been mapped to an approximate 10-cM region on chromosome 3q13 centered around marker D3S1267.7 Two-point LOD-score analysis of four microsatellite markers that map within an interval encompassing D3S1267 generated significant LOD scores indicative of genetic exclusion. Similarly, previous multipoint analysis of the ETM2 locus determined a 2.18-cM interval critical for disease on chromosome 2p25-p22 flanked by markers D2S2150 and D2S220. ${ }^{9}$ Linkage analysis of the pedigree in this study for markers within the ETM2 critical region failed to meet criteria for genetic exclu- 
Table 1

Two-point LOD score analysis reflecting linkage between familial autosomal dominant essential tremor and known disease loci in family

\begin{tabular}{llrrrrrrrrr}
\hline & & \multicolumn{7}{c}{ Recombination fraction $\theta$} \\
\cline { 3 - 9 } Marker & Locus & 0.00 & 0.01 & 0.02 & 0.03 & 0.04 & 0.05 & 0.10 & 0.20 \\
\hline D3S1278 & ETM1 & -3.86 & -2.97 & -2.47 & -2.12 & -1.85 & -1.64 & -0.95 & -0.32 & -0.06 \\
D3S1558 & ETM1 & -2.67 & -2.05 & -1.70 & -1.46 & -1.27 & -1.12 & -0.66 & -0.24 & -0.04 \\
D3S3515 & ETM1 & -5.38 & -4.21 & -3.55 & -3.08 & -2.71 & -2.42 & -1.48 & -0.59 & -0.17 \\
D3S1267 & ETM1 & -4.23 & -3.36 & -2.86 & -2.49 & -2.20 & -1.96 & -1.17 & -0.39 & -0.04 \\
D2S2233 & ETM2 & -4.88 & -3.98 & -3.46 & -3.08 & -2.78 & -2.53 & -1.67 & -0.73 & -0.26 \\
D2S2150 & ETM2 & -0.73 & -0.34 & -0.16 & -0.05 & 0.02 & 0.08 & 0.22 & 0.28 & 0.23 \\
D2S2342 & ETM2 & 0.54 & 0.52 & 0.50 & 0.48 & 0.47 & 0.45 & 0.36 & 0.24 & 0.17 \\
D2S2221 & ETM2 & -4.99 & 2.54 & -2.04 & -1.72 & -1.48 & -1.28 & -0.66 & -0.02 & 0.22 \\
D2S2168 & ETM2 & -7.06 & -4.75 & -3.97 & -3.43 & -3.01 & -2.67 & -1.54 & -0.44 \\
D4S230 & LBP & -5.67 & -4.90 & -4.39 & -3.99 & -3.66 & -3.38 & -2.28 & -1.01 & -0.01 \\
D4S1609 & LBP & 0.39 & 0.38 & 0.36 & 0.34 & 0.33 & 0.32 & 0.26 & 0.17 \\
\hline
\end{tabular}

sion. However, further linkage and haplotype analysis of markers spanning a 5.37-cM region of chromosome 2 encompassing the ETM2 locus indicated that the disease in this family did not map to this region. Marker D2S2168, which maps $2.65 \mathrm{cM}$ centromeric of the critical region, gave a LOD score of -7.06 , with an exclusion interval of approximately $7.5 \mathrm{cM}$. Marker D2S2233, mapping $0.54 \mathrm{cM}$ telomeric of the disease locus, generated a LOD score -4.88 with an approximately 8 -cM region of exclusion. In addition, a common disease haplotype of chromosome $2 p$ markers could not be assigned to all affected individuals (Fig. 1). The gene for LBP, which has been mapped to chromosome 4p between markers D4S1609 and D4S230, ${ }^{10}$ was excluded as a candidate locus with a LOD score of -5.67 for marker D4S230.

Higgins et al. ${ }^{8}$ reported linkage to 2p22-p25 in a large American family of Czech descent with ET and in three other American families. ${ }^{9}$ Gulcher et al. ${ }^{7}$ reported linkage to $3 \mathrm{q} 13$ in 16 Icelandic families with 75 affected individuals. These loci were excluded in our family, suggesting yet another genetic locus we propose to call ETM3, for autosomal dominant ET. Phenotypically, the affected members of our family exhibited classic ET, and in this regard, our family did not differ clinically from the three families studied by Higgins et al..$^{8,9}$ and the 16 families studied by Gulcher et al. ${ }^{7}$ Therefore, the genetic heterogeneity of ET is not reflected in the phenotypes of different families. Earlier age of onset was also noted by Higgins et al. ${ }^{9}$; however, this clinical anticipation may be related to ascertainment bias.

Identification of the genes responsible for ET is critical for elucidating the underlying biochemical defect in the central nervous system and should ultimately lead to the development of successful drug or genetic therapy.

\section{Acknowledgment}

We thank the family for their active participation in this study. This work was funded by the Spastic Paralysis Research
Foundation of Kiwanis International, Illinois-Eastern Iowa District, the Muscular Dystrophy Association, and by the Harriss Malan family.

\section{References}

1. Hubble JP, Busenbark KL, Koller WC. Essential tremor. Clin Neuropharmacol 1989; $12: 453-482$

2. Louis ED, Ottman R. How familial is familial tremor? The genetic epidemiology of essential tremor. Neurology 1996;46:1200-1205.

3. Louis ED, Ottman R, Hauser WA. How common is the most common adult movement disorder? Estimates of the prevalence of essential tremor throughout the world. Mov Disord 1998;13:5-10.

4. Elble RJ. Diagnostic criteria for essential tremor and differential diagnosis. Neurology 2000;54:S2-S6.

5. Schuurman PR, Bosch DA, Bossuyt PM, Bonsel GJ, van Someren EJ, de Bie RM, Merkus MP, Speelman JD. A comparison of continuous thalamic stimulation and thalamotomy for suppression of severe tremor [see comments]. N Engl J Med 2000; 342:461-468.

6. MIM (Online Mendelian Inheritance in Man) Baltimore: Johns Hopkins University, Center for Medical Genetics, January 2001; http://www3.ncbi.nlm.nih.gov/ omim/

7. Gulcher JR, Jonsson P, Kong A, Kristjansson K, Frigge ML, Karason A, Einarsdottir IE, Stefansson H, Einarsdottir AS, Sigurthoardottir S, Baldursson S, Bjornsdottir S, Hrafnkelsdottir SM, Jakobsson F, Benedickz J, Stefansson K. Mapping of a family with essential tremor gene, FET1, to chromosome 3q13. Nat Genet 1997;17:84-87.

8. Higgins JJ, Pho LT, Nee LE. A gene (ETM) for essential tremor maps to chromosome 2p22-p25. Mov Disord 1997;12:859-869.

9. Higgins JJ, Loveless JM, Jankovic J. Evidence that a gene for ET maps to chromosome 2p in 4 families. Mov Disord 1998;13:972-977.

10. Farrer M, Gwinn-Hardy K, Muenter M, DeVrieze FW, Crook R, Perez-Tur J, Lincoln S, Maraganore D, Adler C, Newman S, MacElwee K, McCarthy P, Miller C, Waters C, Hardy J. A chromosome 4p haplotype segregating with Parkinson's disease and postural tremor. Hum Mol Genet 1999;8:81-85.

11. Illarioshkin SN, Ivanova-Smolenskaya IA, Rahmonov RA, Markova ED, Stevanin G, Brice A. Clinical and genetic study of familial essential tremor in an isolate of northern Tajikistan. Mov Disord 2000;15:1020-1023.

12. Bain P, Brin M, Deuschl G, Elble R, Jankovic J, Findley L, Koller WC, Pahwa R. Criteria for the diagnosis of essential tremor. Neurology 2000;54:S7.

13. Amersham Pharmacia Biotech protocol for 5 '-oligolabelling for fluorescence (cata$\log$ \#RPN5755).

14. Lathrop GM, Lalouel JM, Julier C, Ott J. Strategies for multilocus linkage analysis in humans. Proc Natl Acad Sci U S A USA 1984;81:3443-3446. 